\title{
Consideraciones al aplicar los principios de la Excelencia Operacional a la Innovación en el Diseño de Productos
}

\author{
Duran-Novoa, Roberto ${ }^{a}$; Tijerina-Aguilera, Jacobob \\ a Profesor Investigador, División de Extensión, Consultoría e Investigación, Universidad de \\ Monterrey, México; roberto.duran@udem.edu \\ ${ }^{\mathrm{b}}$ Director, División de Extensión, Consultoría e Investigación, Universidad de Monterrey, México; \\ jacobo.tijerina@udem.edu
}

\begin{abstract}
Resumen
Nuestro artículo estudia como las iniciativas orientadas hacia la Calidad y mejora continua (e.g. Six Sigma, TPS-Lean, Operational Excellence) pueden apoyar el desarrollo de la innovación sistemática, basándonos en la hipótesis que las herramientas y buenas prácticas de la Calidad han alcanzado su madurez luego de desarrollarse desde los años 40, situación que no sucede aún con la Innovación y sus principios.
\end{abstract}

Para obtener tendencias generales, estudiamos primero la evolución de las publicaciones asociadas a los términos "calidad" e "innovación" desde 1990 a 2016, para luego enfocar el estudio hacia la compatibilidad entre los principios de excelencia operacional (ExOp) e Innovación en el diseño de productos (IDP). Inicialmente pudimos observar que los estudios acerca de Calidad se desarrollan a un ritmo que disminuye su tasa de crecimiento en los últimos años, apoyando la suposición de maduración. Esto no ocurre con los estudios acerca de Innovación, los cuales mantienen una tasa de crecimiento sostenida, lo que hace razonable suponer que la investigación relacionada a Innovación alcanzará y eventualmente sobrepasará en cantidad a la investigación relacionada a Calidad. Si bien pudimos observar que la ExOp e IDP tienen objetivos generales compatibles, descubrimos que no es recomendable aplicar directamente los principios de la ExOp a la IDP, tanto por motivos empíricos como conceptuales. Dentro de los motivos conceptuales, destacan los conflictos generados por la orientación hacia la eficiencia de la ExOp y la orientación hacia la novedad de la IDP. Esto hace que la IDP promueva la toma de riesgos en búsqueda de la novedad y la anticipación disruptiva, en circunstancias que la ExOp promueve la optimización y propagación de lo validado a través de la experiencia, buscando mejoras incrementales en los procesos. 
Para poder lidiar con las incompatibilidades entre ExOp e IDP, se comparan los principios fundamentales de ambos enfoques y se clasifican en 3 categorias: alta probabilidad de compatibilidad, alta probabilidad de incompatibilidad, y principios que requieren mayor investigación para ser aplicados. Basados en lo anterior, proponemos una lista de principios de ExOp recomendables para quienes se enfoquen en la IDP, de manera que puedan ser un apoyo para desarrollar un proceso robusto, evitando a la vez futuras incompatibilidades. Finalmente, posibles direcciones de investigación son sugeridas, buscando fortalecer la sincronización entre la ExOp y la IDP.

Palabras clave: Calidad, excelencia operacional, innovación, diseño.

\footnotetext{
Abstract

Our article studies how initiatives oriented towards Quality and continuous improvement (e.g. Six Sigma, TPS-Lean, Operational Excellence) can support the development of systematic innovation, based on the hypothesis that the tools and good practices of Quality have reached their maturity after developing since the 40's, which does not happen yet with Innovation and its principles.
}

To obtain general trends first, we study the evolution of the publications associated with the terms "quality" and "innovation" from 1990 to 2016 and then focus on the compatibility between the principles of operational excellence (ExOp) and Product-design innovation (IDP.) Initially, we observed that Quality related studies development rate is decreasing through time, supporting the assumption of maturity. This does not happen with Innovation related studies which are growing over time, making reasonable to assume that Innovation related research will reach and eventually surpass in quantity the Quality related research. Although we could observe that the ExOp and IDP have compatible general objectives, we discovered that it is not advisable to apply directly the principles of ExOp to the IDP, both for empirical and conceptual reasons. Within the conceptual reasons, stand out the potential conflict between the efficiency-orientation of the ExOp and the novelty-orientation of the IDP. This makes the IDP prone to taking risks in search of novelty and disruptive anticipation, in circumstances that the ExOp 
promotes the optimization and propagation of what has been validated through experience, seeking incremental improvements in the processes.

In order to deal with the incompatibilities between ExOp and IDP, the fundamental principles of both approaches are compared and classified into 3 categories: high compatibility probability, high incompatibility probability, and principles that require further investigation to be classified. Based on the previous, we propose a list of recommendable ExOp principles for those who focus on the IDP, in order to develop a robust process while avoiding future incompatibilities. Finally, possible research directions are suggested, seeking to strengthen the synchronization between ExOp and IDP.

Keywords: Quality, operational excellence, innovation, design.

\section{Introducción}

Los sistemas evolucionan, y el estudio de esta evolución ha permitido mejorar su entendimiento. Durante el siglo XX, el estudio de sistemas industriales estuvo centrado en la productividad, a fines del siglo XX se centró en la Calidad, y actualmente, basándose en el conocimiento anterior, es la Innovación quien concentra la atención. Será -al parecer- el conocimiento que desarrollemos respecto a ella quien determine nuestra capacidad de adaptarnos al cambio, y por ende, al diseño.

Considerando lo anterior, nuestro artículo estudia como las iniciativas orientadas hacia la Calidad y mejora continua (e.g. Six Sigma, TPS-Lean, Operational excellence) pueden apoyar el desarrollo de la Innovación sistemática. Nuestra hipótesis es que las herramientas y buenas prácticas de la Calidad están alcanzado su madurez luego de desarrollarse desde los años 40, situación que no sucede aún con la Innovación y sus principios respectivos (Fagerberg, Martin, \& Andersen, 2013; Lee, Kao, \& Yang, 2014; Sridharan, 2004; Stamm, Neitzert, \& Singh, 2009; Wilbur, 2002; Xiaofen, 2013).

\subsection{Innovación}

La Innovación ha sido relacionada constantemente con el desarrollo de productos y su proceso, sin embargo, en el último tiempo su marco de medición se ha extendido de tres maneras importantes. En primer lugar, haciendo hincapié en el papel de los vínculos con otras empresas e instituciones durante su desarrollo; en segundo lugar, reconociendo la 
importancia de las empresas con investigación y desarrollo (I+D) intensivo; y en tercer lugar, incluyendo los desarrollos en las áreas de organización y marketing. Tomando como base la definición del Manual de Oslo, podemos decir que una Innovación es la implementación de un nuevo o significativamente mejorado producto (bien o servicio), proceso, método de marketing, o método organizacional (prácticas comerciales, organización laboral, o relaciones externas) (OCDE, 2005). Las innovaciones no necesariamente se convierten en un éxito comercial -muchas de ellas fracasan- sin embargo, su razón última es mejorar el rendimiento de la empresa innovadora. Existen muchos factores que influyen en la Innovación, principalmente económicos, (e.g. altos costos, falta de demanda), legales (e.g. regulaciones, normas fiscales), y relacionados a las características de cada empresa (e.g. personal calificado, cultura).

Algunas de sus variables principales son:

- Incertidumbre: no es posible saber de antemano cuál será el resultado de la Innovación, ni cuántos recursos se necesitarán para implementar un nuevo proceso.

- La apropiación: los costos de desarrollo suelen ser mucho mayores que la puesta en marcha, por lo que los competidores pueden beneficiarse más que quien desarrolla.

- Inversión: generalmente requiere la adquisición de activos fijos e intangibles, junto a la realización de otras actividades orientadas a rentabilidades futuras.

- Efectos indirectos: las empresas innovadoras pueden beneficiarse de los efectos del conocimiento o del uso de la Innovación original (ej. vía patentes).

- Alejamiento de las rutinas: el uso de nuevos conocimientos o la combinación de conocimientos existentes requiere trabajar lejos de rutinas estandarizadas.

- Ventaja competitiva: ya sea cambiando la curva de demanda, ofreciendo nuevos productos, o mejorando la capacidad de la empresa.

Una empresa puede participar en muchas actividades innovadoras que no son necesariamente de $\mathrm{I}+\mathrm{D}$, como reformular las relaciones con los clientes, estudiar las estrategias ajenas, utilizar consultores para proyectos específicos, comprar información técnica, pagar por invenciones patentadas, invertir en equipos o software, etc. Sin embargo, hay cambios que escapan del concepto de Innovación, por ejemplo:

- Dejar de utilizar un proceso, un método de marketing, un método organizacional, o dejar de comercializar un producto.

- Aumentar el capital de la organización.

- Aplicar cambios en los precios de los insumos al producto. 
- Personalizar un producto para un número limitado de clientes, sin incluir atributos significativamente diferentes al estándar.

- Realizar cambios estacionales u otros cambios cíclicos (e.g. vestimenta operarios).

- Comerciar productos nuevos o mejorados (reventa).

- Diseñar cambios que no alteran la función, el uso previsto o las características técnicas de un bien o servicio.

En resumen, puede notarse que los requisitos esenciales de la Innovación son la novedad y su implementación, independiente del área de aplicación.

\subsection{Tendencias relacionadas a la investigación de la Calidad y la Innovación}

Para obtener tendencias generales, estudiamos primero la evolución de las publicaciones asociadas a los términos "calidad" e "innovación” desde 1990 a 2016 a través de la plataforma SCOPUS. De ser necesario, estos resultados serán acotados hacia la compatibilidad entre los principios de Excelencia Operacional (ExOp) e Innovación en el Diseño de Productos (IDP). En las Figuras 1 y 2, puede apreciarse como la Calidad se encuentra alcanzando su madurez, mientras que la Innovación está acelerando su crecimiento, acercándose a la producción en Calidad. Puesto que ambas temáticas son fundamentales para el desarrollo de productos, es esperable que la Innovación pueda beneficiarse de los estudios realizados en las áreas de Calidad, y que en un futuro cercano la Calidad sea considerada como un requisito esencial de las empresas, tal como hoy sucede con la Productividad.

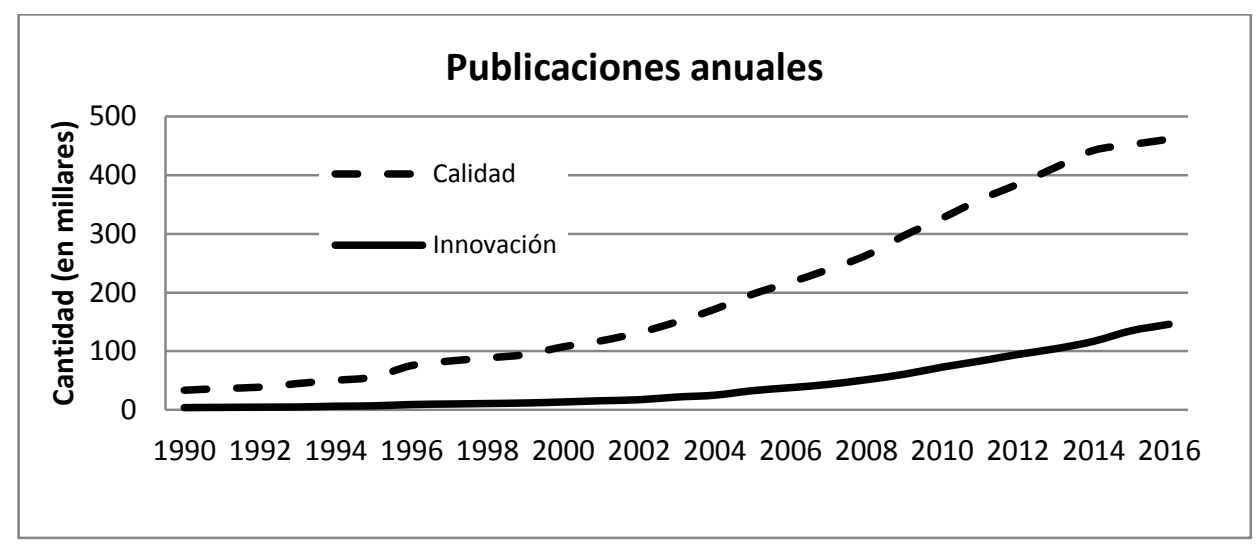

Fig. 1 Evolución de las publicaciones anuales relacionadas a Calidad en Innovación, escala lineal 


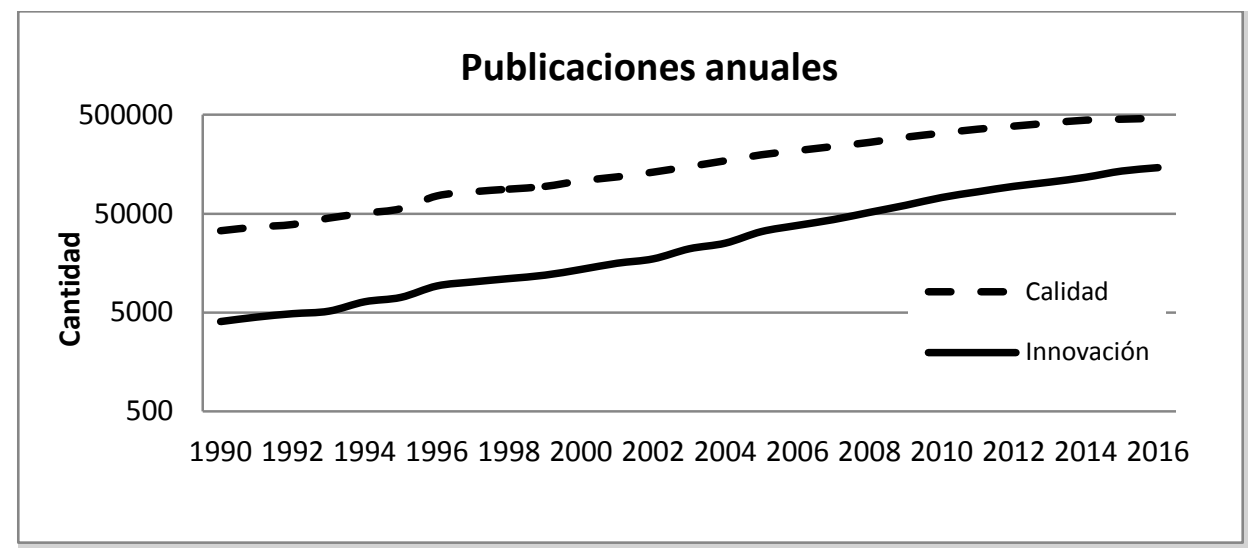

Fig. 2 Evolución de las publicaciones anuales relacionadas a Calidad en Innovación, escala logaritmica.

Para poder comparar que principios de las iniciativas de Calidad son probablemente compatibles con los requisitos de la Innovación y cuales requieren mayor análisis, la siguiente sección presenta un resumen de 5 iniciativas reconocidas en el área de la Calidad, en base a sus principios fundamentales.

\section{Iniciativas de Calidad y Excelencia Operacional}

\subsection{Six Sigma}

Six Sigma se basa en la implementación rigurosa de principios y técnicas de Calidad probadas, por medio de un pequeño grupo de líderes ("Black-belts"). Lo anterior tiene como objetivo principal un funcionamiento sin errores, gracias a la toma de decisiones basadas en datos en todos los niveles de la organización, centrada en los beneficios de sus tres grupos de interesados: clientes, accionistas y empleados (Pyzdek \& Keller, 2010). Un programa Six Sigma correctamente implementado debe poseer las siguientes características (adaptadas de (Juran \& Godfrey, 1998; Keller, 2011)):

I. Orientación al cliente: Vinculada al reto de relacionar la satisfacción de los clientes con su comportamiento real, idealmente creando negocios sin perder clientes.

II. Valor de todos los asociados. Los empleados pueden aportar directamente con ideas (e.g. Toyota implementa más de 46 ideas/empleado por año), siendo indispensables en la creación de una cultura capaz de adaptarse y perdurar.

III. Mejora continua. La mejora implica la satisfacción del cliente, y por tanto adaptarse al cambio. 
IV. Apoyo ejecutivo. Los proyectos son liderados por el equipo ejecutivo, que utilizan Six Sigma para promover sus metas. Los "Six Sigma Champions", asignan recursos a los equipos de proyecto (autoridad, difusión, etc.) en pos del éxito.

V. Infraestructura. Los equipos están dirigidos por los Black-belts, capacitados a tiempo completo en el área de análisis estadístico y resolución de problemas, mientras que el personal entrenado en los métodos básicos, los Green-belts, se enfocan en los procesos.

VI. Metodología. La metodología estándar DMAIC (Define-Measure-AnalyzeImprove-Control) asegura que los proyectos Six Sigma estén claramente definidos e implementados, y que la toma de decisiones se sustente en datos, evitando así mejoras parciales o de corta duración.

VII. Entrenamiento. Una vez que los Champions han sido entrenados y se han establecido criterios de selección de proyectos, se entrenan los Black-belts en la aplicación de DMAIC, para luego seleccionar los Green-belts de las áreas críticas del proceso.

\subsection{Control Total de Calidad}

El Control Total de Calidad (TQC) es la versión japonesa -y evolucionada- del sistema homónimo estadounidense, basado en seis principios (Ishikawa, 1985; Pyzdek \& Keller, 2013):

I. La calidad primero (no los beneficios a corto plazo). Esta mentalidad puede ganar la confianza del cliente paso a paso, lo que resulta en un aumento gradual en las ventas de la empresa, generando mejoras a largo plazo en la rentabilidad y la estabilidad de gestión. Pensamientos a corto plazo pueden obtener retornos más rápidos, pero serán incapaces de mantener la competitividad por períodos extendidos.

II. Orientación al consumidor (no al productor). Se debe pensar desde el punto de vista de los otros involucrados, respetándolos con atención.

III. Objetivos transversales (no departamentales). La empresa en su conjunto debe estudiar sus procesos globalmente buscando entregar el valor al cliente, en lugar de enfocarse en un departamento o sección en particular.

IV. Basarse en hechos y métodos estadísticos (no opiniones). Los hechos pueden traducirse en datos, los cuales pueden analizarse estadísticamente para guiar la toma de decisiones de acuerdo a los objetivos de la administración.

V. Respeto por la humanidad como filosofía de gestión. El principio fundamental de una gestión exitosa es permitir que los subordinados hagan pleno uso de su capacidad, otorgándoles autonomía y espontaneidad, para que su potencial pueda desarrollarse. 
VI. Gestión interfuncional. La empresa debe establecer comités transversales para abordar sus funciones principales (garantía de calidad, control de costos, control de cantidad y control del personal). El presidente del comité debe ser un director senior, y los otros miembros deben seleccionarse de altos cargos de los distintos departamentos.

\subsection{ISO 9000: 2000}

La revisión de ISO 9000: 2000, se enfocó en el control del proceso y la mejora continua a través de una visión más sistemática que sus versiones anteriores. En ella se enumeran ocho principios de gestión de la calidad (Juran \& Godfrey, 1998; Stapp, 2001):

I. Enfoque en el cliente. El intento continuo de satisfacer los requerimientos y superar las expectativas de los clientes debe ser un objetivo fundamental de toda organización.

II. Liderazgo. Es necesario para generar un ambiente adecuado que permita dirigirse hacia los objetivos de la organización.

III. Participación del personal. El liderazgo debe aprovechar los talentos de toda la organización en el logro de sus objetivos. Esto hace necesario una interacción transversal que involucre a todo el personal.

IV. Enfoque en el proceso. Todo lo que una organización hace debe considerarse un proceso lógico, considerando insumos, recursos, y el objetivo deseado.

V. Enfoque sistémico de la gestión. Sólo cuando la organización identifica y administra los distintos procesos y sus relaciones, se pueden cumplir los objetivos.

VI. Mejora continua. Toda organización debe comprometerse a mejorar en todas sus actividades (mayor eficiencia, menor rechazo, clientes más satisfechos, etc.).

VII. Toma de decisiones basada en hechos. La organización debe recolectar información sobre sus sistemas, y usarlos como la base que asegure la satisfacción del cliente y la mejora continua.

VIII. Relaciones beneficiosas con el proveedor. Dado que los proveedores forman parte integral de los sistemas que una organización debe gestionar, la creación de una relación de cooperación con ellos debe ser valorada.

\subsection{Principios Toyota}

Los principios Toyota (TPS) han sido estudiados y aplicados en muchos contextos. Nosotros usaremos como referencia el estudio de (Liker \& Morgan, 2006), el cual incluye modificaciones que facilitan la aplicación de los principios Toyota -y Lean en general- más allá de contextos técnicos, en sintonía con los objetivos de la Excelencia Operacional. Este enfoque considera que existen 13 principios de gestión fundamentales que deben ser aplicados constantemente en búsqueda de una cultura organizacional, los cuales se sub- 
clasifican en las categorías de Procesos (principios I a IV), Personas (principios V a X), y Herramientas-tecnología (principios XI a XIII). Los principios son:

I. Establecer valor en base al cliente, separando valor agregado de residuos. Lean es una eliminación interminable de residuos, los cuales son determinados en base al cliente.

II. Enfrentar el proceso de desarrollo de productos cuando exista mayor espacio de diseño, explorando a fondo soluciones alternativas. Definir el problema equivocado o enfocarse prematuramente en la solución equivocada tendrá costos a lo largo de todo el ciclo de vida del producto. Explorar a fondo las alternativas enfocándose en la raíz de los problemas -idealmente anticipándose- tiene beneficios exponenciales.

III. Crear un flujo de Proceso de Desarrollo de Productos nivelado. Inicialmente debe estabilizarse el proceso, para que sea predecible y planificable; esto permite la reducción de oscilaciones bruscas en la carga de trabajo. Las oscilaciones estándar pueden ser atendidas a través de grupos de trabajo flexibles, además de traer problemas potenciales a la superficie.

IV. Utilizar una normalización rigurosa que reduzca la variación, aumentado la flexibilidad y predictibilidad. La normalización es la base para la mejora continua, y la estandarización del producto y el proceso son la base para todos los otros principios.

V. Desarrollar un "Sistema de Ingenieros Jefe" para integrar el desarrollo de inicio a fin. El ingeniero jefe tiene la autoridad y responsabilidad final de todo el proceso de desarrollo del producto, siendo la principal fuente de integración de productos y procesos.

VI. Organizar equilibrando la expertiz funcional y la integración multifuncional. La experiencia funcional combinada con objetivos transversales proporcionan el equilibrio buscado por la organización matricial.

VII. Desarrollar una competencia técnica elevada en todos los ingenieros. Los ingenieros deben tener un conocimiento especializado del producto y el proceso, desarrollados en base a la experiencia directa.

VIII. Integrar a los proveedores al sistema de desarrollo de productos. Los proveedores deben poder integrarse al proceso de desarrollo, en base a capacidades y cultura compatibles.

IX. Construir Aprendizaje y Mejora Continua interna. El aprendizaje organizacional es una condición necesaria para la mejora continua y se basa en todos los demás principios.

$X$. Construir una cultura que apoye la excelencia y la mejora implacable. La excelencia y la mejora son un reflejo de la cultura organizacional y por lo tanto deben estar presentes en cada acción de la organización. 
XI. Adaptar la tecnología en función de las personas y el proceso. La tecnología debe subordinarse a las personas y el proceso, no viceversa.

XII. Alinear la organización usando una comunicación visual sencilla. Los objetivos deben estar alineados transversalmente, y toda la organización debe poder aportar mediante una comunicación visual simple.

XIII. Utilizar herramientas potentes para normalizar y apoyar el aprendizaje organizacional. Herramientas potentes y sencillas pueden generar la normalización necesaria para el aprendizaje organizacional.

\subsection{Shingo model (excelencia operacional)}

La excelencia operacional (ExOp) es la visión que muchas organizaciones han establecido para impulsar la mejora. Programas, nombres, herramientas, proyectos y personalidades son insuficientes para crear un cambio duradero. Un cambio real sólo es posible cuando los principios intemporales de la excelencia operacional se comprenden y se insertan profundamente en la cultura. El modelo Shingo para la ExOp ha sido desarrollado por el Shingo Institute, a partir de la investigación de Shigeo Shingo (ver http://www.shingoprize.org/). Plantea que existen 10 principios rectores de la ExOp, los cuales se agrupan en cuatro dimensiones: facilitadores culturales (principios I a II), mejora continua de procesos (principios III a VII), alineación de empresas (principios VIII y IX), y resultados (principio X). Grandes resultados son el efecto de seguir los principios que rigen los resultados, en consecuencia, para obtener resultados ideales se necesita un comportamiento ideal; esto es lo que debe entenderse por ExOp (Miller, Raymer, Cook, \& Barker, 2013). Los 10 principios son:

I. Liderar con humildad. Permite estar abierto a buenas ideas e innovación desde cualquier parte de la organización. Liderar con humildad permite aceptar la responsabilidad y realizar los cambios.

II. Respetar a cada individuo. Permite poner la seguridad primero, capacitar a las personas, y dejarlas actuar independientemente. El respeto permite que todas las comunicaciones clave estén abiertas.

III. Enfocarse en el proceso. Cualquier producto es creado por procesos que actúan sobre entradas. Los buenos procesos producen los productos deseados, siempre y cuando se proporcionen los insumos apropiados. Este enfoque ayuda a concentrar la resolución de problemas en el proceso y no en las personas (eliminando las búsquedas de "culpables"), creando un ambiente que facilita el aprendizaje.

IV. Utilizar el pensamiento científico. Además de acoplarse al enfoque en el proceso, el pensamiento científico se basa en los resultados, lo que hace prioritario definir y comunicar los resultados deseados a través de la organización. Modelos como el PDCA (Plan, Do, Check, Act) o DMAIC (ver Six sigma) pueden ser utilizados. 
V. Utilizar el valor del "flujo" y la "tracción". El pensamiento de flujo busca acortar el tiempo de entrega desde el inicio, eliminando las barreras (residuos) que impiden la creación de valor y entrega al cliente. La "tracción" busca equiparar la tasa de producción al nivel de demanda. Ambos se necesitan y crean beneficios transversales.

VI. Asegurar la calidad en la fuente. Implica (1) no permitir que defectos avancen, (2) detener y solucionar problemas y (3) respetar al individuo en el proceso.

VII. Buscar la perfección. La mejora continua del proceso no tiene fin. La búsqueda de la perfección revela que siempre hay oportunidades de mejora. La solución de problemas puede implicar que después de una solución se realice una mejora.

VIII. Crear un propósito permanente. Las organizaciones están en constante cambio: sus clientes, expectativas, competidores, mercados, la tecnología, los liderazgos, procesos, productos, estrategias, incluso los valores de una empresa pueden cambiar. Aun así, es necesario determinar el por qué existe cada organización, cuál es su propósito. Este sentido de la dirección ayuda a no perder el rumbo, por ejemplo cuando las decisiones tácticas requieran desvíos temporales. Las organizaciones que frecuentemente redirigen filosofías y estrategias son incapaces de cuantificar el desperdicio asociado a la inestabilidad, la fluctuación, y la pérdida de compromiso humano.

IX. Pensar sistemáticamente. Permite unificar los demás principios de ExOp, demandando a las organizaciones análisis y síntesis. A medida que los líderes se mueven hacia el pensamiento sistémico, la ExOp puede aplicarse transversalmente en la organización.

X. Crear valor para el cliente. Todo aspecto de una organización debe centrarse en la creación de valor para el cliente, siendo la guía base para la toma de decisiones y la mejora continua. Una organización debe impulsar todo lo que aumente el valor, incluyendo calidad, costo, entrega, seguridad, y moral.

\section{Análisis de compatibilidad entre iniciativas de Calidad e Innovación}

Antes de realizar cualquier análisis es importante recordar que los requisitos esenciales de la Innovación son la novedad -o mejora significativa- y su implementación, independiente del área de aplicación. No puede existir Innovación si no se cumplen estas condiciones.

Otros estudios han analizado los posibles conflictos entre iniciativas, concluyendo que cada empresa en particular deberá decidir cuál iniciativa implementar basándose en sus características particulares. Esto se debe a que cada iniciativa conduce hacia conceptos comunes, partiendo desde diferentes puntos de vista (muchos efectos secundarios de una iniciativa se asemejan a los principios de enfoque principal de otra iniciativa) (Nave, 2002). 
Sin embargo, a la fecha no se han desarrollado criterios objetivos bajo los cuales comparar iniciativas. Considerando los principios fundamentales de las iniciativas estudiadas en la Sección 2, la Tabla 1 presenta un resumen de la compatibilidad entre estas iniciativas y las necesidades de la Innovación. En ella, los principios de las distintas iniciativas han sido alineados por afinidad, entendiendo que, si bien no son equivalentes, pueden ser comparados simultáneamente con los requerimientos de la Innovación pues apuntan en la misma dirección (confirmando lo planteado por (Nave, 2002)). La columna "Relación" presenta el tipo de relación más probable entre cada principio y la Innovación bajo la siguiente lógica:

- La letra "C" indica que existe una alta probabilidad de compatibilidad.

- La letra "I" indica que existe una alta probabilidad de incompatibilidad.

- La combinación "I-C" indica que el contexto será el principal determinante del tipo de relación, por lo que el estudio de estos principios requiere un análisis más profundo para obtener directrices.

Tabla 1. Principios de las iniciativas (resumidos) y su relación más probable con la Innovación

\begin{tabular}{|c|c|c|c|c|c|}
\hline Six Sigma & TQC & ISO 9000: 2000 & TPS & Shingo & Relación \\
\hline \multirow[t]{2}{*}{$\begin{array}{l}\text { - Orientación al } \\
\text { cliente }\end{array}$} & $\begin{array}{l}\text { - Orientación al } \\
\text { consumidor }\end{array}$ & $\begin{array}{l}\text { - Enfoque en el } \\
\text { cliente }\end{array}$ & $\begin{array}{l}\text { - Valor en base al } \\
\text { cliente }\end{array}$ & $\begin{array}{l}\text { - Crear valor para el } \\
\text { cliente. }\end{array}$ & $\mathrm{C}$ \\
\hline & & & $\begin{array}{l}\text { - Alinear a la } \\
\text { organización }\end{array}$ & - Crear un propósito & $\mathrm{C}$ \\
\hline $\begin{array}{l}\text { - Valor de los } \\
\text { asociados. }\end{array}$ & $\begin{array}{l}\text { - Respeto por la } \\
\text { humanidad }\end{array}$ & $\begin{array}{l}\text { - Participación } \\
\text { del personal }\end{array}$ & & - Respetar a todos & $\mathrm{C}$ \\
\hline $\begin{array}{l}\text { - Apoyo } \\
\text { ejecutivo }\end{array}$ & $\begin{array}{l}\text { - Objetivos } \\
\text { transversales }\end{array}$ & - Liderazgo & $\begin{array}{l}\text { - Equilibrar integración } \\
\text { y experiencia. }\end{array}$ & - Liderazgo humilde & $\mathrm{C}$ \\
\hline \multirow[t]{2}{*}{ - Infraestructura } & & & $\begin{array}{l}\text { - Alta competencia } \\
\text { técnica }\end{array}$ & - Pens. científico & $\mathrm{C}$ \\
\hline & & & $\begin{array}{l}\text { - Desarrollar cuando } \\
\text { exista mayor espacio }\end{array}$ & & $\mathrm{C}$ \\
\hline \multirow[t]{2}{*}{ - Mejora continua } & & - Mejora continua & $\begin{array}{l}\text { - Aprendizaje y mejora } \\
\text { continua }\end{array}$ & - Buscar la perfección & I \\
\hline & - Calidad primero & & - Cultura de excelencia & - Flujo y tracción & I \\
\hline \multirow[t]{3}{*}{ - Entrenamiento } & $\begin{array}{l}\text { - Gestión } \\
\text { interfuncional }\end{array}$ & & $\begin{array}{l}\text { - Aprendizaje org. y } \\
\text { normalizar }\end{array}$ & & I \\
\hline & & $\begin{array}{l}\text { - Enfoque en el } \\
\text { proceso }\end{array}$ & - Proceso nivelado & $\begin{array}{l}\text { - Enfoque en el } \\
\text { proceso }\end{array}$ & I \\
\hline & & & $\begin{array}{l}\text { - Adaptar tecno. a las } \\
\text { personas y los proceso }\end{array}$ & & I \\
\hline \multirow[t]{3}{*}{ - Metodología } & $\begin{array}{l}\text { - Basarse en } \\
\text { estadisticas }\end{array}$ & $\begin{array}{l}\text { - Decisiones } \\
\text { basada en hechos }\end{array}$ & - Sistema de Ing. Jefe & & $\mathrm{I}-\mathrm{C}$ \\
\hline & & $\begin{array}{l}\text { - Gestión } \\
\text { sistémica }\end{array}$ & - Reducir la variación & - Pens. sistemático & $\mathrm{I}-\mathrm{C}$ \\
\hline & & $\begin{array}{l}\text { - Relacionarse } \\
\text { con el proveedor }\end{array}$ & $\begin{array}{l}\text { - Integrar a los } \\
\text { proveedores }\end{array}$ & $\begin{array}{l}\text { - Asegurar la calidad } \\
\text { en la fuente }\end{array}$ & $\mathrm{I}-\mathrm{C}$ \\
\hline
\end{tabular}


Es posible observar que muchos principios fundamentales de la Calidad y ExOp tienen una alta probabilidad de incompatibilidad, en algunos casos no tan evidente. Por ejemplo, siguiendo el orden de la tabla, la mejora continua puede generar serios inconvenientes en un ambiente que busca innovar: es muy probable que el aplicar técnicas validadas o buscar la perfección de manera constante y gradual tenga un efecto negativo al trabajar en entornos donde se requiere desarrollar y aplicar la novedad y cambios significativos; adicionalmente, la generación de residuos al innovar es inevitable. Un proceso que busque "traccionar" Innovación probablemente genere iniciativas incompletas, las cuales pueden permitir a la competencia observar el producto propio y luego lanzar su versión desarrollada a un menor costo. Si bien la Innovación debiera idealmente ser desarrollada sistemáticamente, su progreso no es continuo como el de la optimización, por lo que enfocarse en el proceso puede desviar la atención de su verdadero propósito (mejores resultados mediante la aplicación de novedad). Por último, es poco probable que la Innovación pueda adaptar la tecnología y los procesos a los empleados, debido a que generalmente el desarrollo de productos novedosos requiere desarrollar tecnologías que se puedan incorporar rápidamente, sin poder esperar a las personalizaciones (una vez obtenidos productos operativos estos pueden ser optimizados y personalizados).

\subsection{Descubrimientos}

Inicialmente, pudimos observar que los estudios acerca de Calidad se desarrollan a un ritmo que se está estabilizando en los últimos años, confirmando la suposición de maduración. Esto no ocurre con los estudios acerca de Innovación, los cuales aumentan su tasa de crecimiento, siendo razonable suponer que la investigación relacionada a la Innovación sobrepasará en cantidad a la realizada acerca de Calidad.

Si bien queda claro que los objetivos generales de la ExOp y la IDP -centrados en el desarrollo de las organizaciones- son compatibles, descubrimos que no es recomendable aplicar directamente los principios de la ExOp a la IDP, tanto por motivos empíricos como conceptuales. Dentro de estos últimos, cobran fuerza los conflictos generados por la naturaleza continua y repetitiva de la ExOp, lo cual limita la compatibilidad debido a la naturaleza anticipativa y disruptiva de la IDP. Podemos decir que lo eficiente de la ExOp no podrá ser aplicado inmediatamente a un proceso innovador, el cual por su esencia novedosa podrá aspirar como máximo a la eficacia, debiendo estar preparado para lidiar con el fracaso.

Finalmente, puede decirse que en general la ExOp es un buen punto de partida para la IDP, no un contexto adecuado para su desarrollo. La IDP debe ser capaz de avanzar a su propio ritmo, incomodando a las áreas que sean necesarias y adaptándose a las circunstancias particulares con tal de desarrollar y aplicar algo novedoso. 


\section{Consideraciones e investigación futura}

Este estudio es el comienzo de una investigación respecto a la evolución de las iniciativas de Calidad y su posible relación con el desarrollo de la Innovación. Puesto que se han seguido líneas generales, los resultados son tendencias aceptables que deben ser validadas por estudios especialmente diseñados (con experimentos adecuados), que permitan determinar los resultados de compatibilidad más probables para cada principio en la práctica del desarrollo organizacional.

\section{Referencias}

Fagerberg, J., Martin, B. R., \& Andersen, E. S. (2013). Innovation studies : evolution and future challenges (1st ed.). Oxford University Press.

Ishikawa, K. (1985). What is Total Quality Control?

Juran, J. M., \& Godfrey, A. B. (1998). Juran's Quality Control Handbook. McGrawHill. https://doi.org/10.1108/09684879310045286

Keller, P. (2011). Six Sigma Demystified, Second Edition. https://doi.org/doi:10.1036/9780071760829

Lee, J., Kao, H. A., \& Yang, S. (2014). Service innovation and smart analytics for Industry 4.0 and big data environment. In Procedia CIRP (Vol. 16, pp. 3-8). Elsevier. https://doi.org/10.1016/j.procir.2014.02.001

Liker, J. K., \& Morgan, J. M. (2006). The Toyota Way in Services: The Case of Lean Product Development. Academy of Management Perspectives, 20(2), 5-20. https://doi.org/10.5465/AMP.2006.20591002

Miller, R., Raymer, J., Cook, R., \& Barker, S. (2013). The Shingo model for operational excellence. Logan, Utah.

Nave, D. (2002). How to Compare Six Sigma, Lean Management, and the Theory of Constraints. Quality Process, 35, 73-78.

OCDE. (2005). Oslo Manual - Guidelines for collecting and interpreting innovation data. Communities (Third, Vol. Third edit). OECD Publishing. https://doi.org/10.1787/9789264013100-en

Pyzdek, T., \& Keller, P. (2010). The Six Sigma handbook. Mc Graw Hill. https://doi.org/10.1036/0071415963

Pyzdek, T., \& Keller, P. (2013). The Handbook for Quality Management A Complete Guide to Operational Excellence. Zhurnal Eksperimental'noi i Teoreticheskoi Fiziki.

Sridharan, E. (2004). Evolving Towards Innovation? The Recent Evolution and Future Trajectory of the Indian Software Industry. In India in the Global Software Industry (pp. 27-50). London: Palgrave Macmillan UK. 
https://doi.org/10.1057/9781403943842_2

Stamm, M. L., Neitzert, T. R., \& Singh, D. P. K. (2009). TQM, TPM, TOC, Lean and Six Sigma-evolution of manufacturing methodologies under the paradigm shift from Taylorism/Fordism to Toyotism. School of Engineering AUT University, (1), 1-10. Retrieved from http://aut.researchgateway.ac.nz/handle/10292/3858

Stapp, E. H. (2001). ISO 9000: 2000: An Essential Guide to the New Standard. Tucson: QA Publishing, LLC.

Wilbur, J. H. (2002). Is time running out for quality? Quality Progress, 35(7), 75-79.

Xiaofen, T. (2013). Investigation on quality management maturity of Shanghai enterprises. The TQM Journal, 25(4), 417-430. https://doi.org/10.1108/17542731311314890 